\title{
CONCERNING AN APPROXIMATION OF COPSON
}

\author{
J. D. BUCKHOLTZ
}

For each complex number $z$ and each positive integer $n$, define $S_{n}(z)$ by the equation

$$
e^{n z}=\sum_{p=0}^{n} \frac{(n z)^{p}}{p !}+\frac{(n z)^{n}}{n !} S_{n}(z) .
$$

In 1913, Ramanujan [2] made the assertion (in a somewhat different notation) that

$$
S_{n}(1)=\frac{n !}{2}\left(\frac{e}{n}\right)^{n}-\frac{2}{3}+\frac{4}{135 n}+O\left(\frac{1}{n^{2}}\right) .
$$

Proofs for this and related matters were given independently in 1928 by G. Szegö [3] and G. N. Watson [4].

In 1932, E. T. Copson [1] proved that $\left\{S_{n}(-1)\right\}$ is a decreasing sequence with limit $-\frac{1}{2}$, and derived the asymptotic series

$$
S_{n}(-1) \sim-\frac{1}{2}+\frac{1}{8 n}+\frac{1}{32 n^{2}}-\frac{1}{128 n^{3}}-\frac{13}{256 n^{4}}+\cdots .
$$

The determination of the coefficients is quite complicated (and, for the coefficient of $n^{-4}$, incorrect).

In the present paper we obtain Copson's series by a simpler method which yields an asymptotic expansion for $S_{n}(z)$ valid for every complex number $z$ except $z=1$.

For our principle result we require the following three lemmas concerning the function $S_{n}(z)$ defined by (1) and the function $T_{n}(z)$ defined by

$$
e^{n z}=\frac{(n z)^{n}}{n !} T_{n}(z)+\sum_{p=n+1}^{\infty} \frac{(n z)^{p}}{p !} .
$$

Lemma 1. For each positive integer $n$, the functions $S_{n}(z)$ and $T_{n}(z)$ have the following properties:

$$
S_{n}(z)+T_{n}(z)=\frac{n !(e / n)^{n}}{\left(z e^{1-z}\right)^{n}}
$$

Received by the editors May 18, 1962. 
(ii)

$$
S_{n}(z)=\frac{z}{1-z}\left[1-\frac{S_{n}^{\prime}(z)}{n}\right]
$$

and

$$
T_{n}(z)=\frac{z}{z-1}\left[1+\frac{T_{n}^{\prime}(z)}{n}\right]
$$

(iii) $\left|S_{n}(z)\right|<e n^{1 / 2}$ if $|z| \leqq 1,\left|T_{n}(z)\right|<e n^{1 / 2}$ if $|z| \geqq 1$; and if $\left|z e^{1-z}\right| \geqq 1$, then both $\left|S_{n}(z)\right|$ and $\left|T_{n}(z)\right|$ are less than $2 e n^{1 / 2}$.

INDICATION OF PROOF. (i) and (ii) are direct consequences of the definitions. To prove (iii) we make use of the fact that

$$
\begin{array}{ll}
\left|S_{n}(z)\right| \leqq S_{n}(1) & \text { if }|z| \leqq 1, \text { and } \\
\left|T_{n}(z)\right| \leqq T_{n}(1) & \text { if }|z| \geqq 1 .
\end{array}
$$

Since each of $S_{n}(1)$ and $T_{n}(1)$ is positive, neither is as large as $S_{n}(1)+T_{n}(1)=n !(e / n)^{n} \leqq e n^{1 / 2}$; the last part of (iii) then follows from (i).

Notation. The linear operator which transforms $f(z)$ into $z f^{\prime}(z) /(z-1)$ will be denoted by $(z /(z-1))(d / d z)$. $J$ and $K$ will be the sets given by $\left\{z:|z| \leqq 1\right.$ and $\left.\left|z e^{1-z}\right| \leqq 1\right\}$ and $\{z:|z| \geqq 1$ and $\left.\left|z e^{1-z}\right| \leqq 1\right\}$, respectively. If $M$ is a set, $d_{M}(z)$ and $\phi_{M}(z)$ will denote the distance and characteristic functions for the set $M$; specifically, $d_{M}(z)$ is the greatest lower bound of distances from $z$ to points of $M$, and $\phi_{M}(z)$ is 1 or 0 according as $z$ is or is not in $M$.

Lemma 2. For every positive integer $k$,

$$
S_{n}(z)=\sum_{r=0}^{k-1}\left(\frac{1}{n} \frac{z}{z-1} \frac{d}{d z}\right)^{r} \frac{z}{1-z}+\left(\frac{1}{n} \frac{z}{z-1} \frac{d}{d z}\right)^{k} S_{n}(z),
$$

and

$$
T_{n}(z)=\sum_{r=0}^{k-1}\left(\frac{1}{n} \frac{z}{z-1} \frac{d}{d z}\right)^{r} \frac{z}{z-1}+\left(\frac{1}{n} \frac{z}{z-1} \frac{d}{d z}\right)^{k} T_{n}(z) .
$$

Proof. Mathematical induction and (ii) of Lemma 1.

Lemma 3. Suppose $0<\epsilon<1$. If $k$ is a nonnegative integer, then

$$
\left|\left(\frac{z}{z-1} \frac{d}{d z}\right)^{k} S_{n}(z)\right|<14 \frac{2^{k^{2}+3 k}}{\epsilon^{2 k+2}} \quad \text { if } d_{K}(z) \geqq \epsilon,
$$

and 


$$
\left|\left(\frac{z}{z-1} \frac{d}{d z}\right)^{k} T_{n}(z)\right|<14 \frac{2^{k^{2}+3 k}}{\epsilon^{2 k+2}} \quad \text { if } d_{J}(z) \geqq \epsilon .
$$

Proof. Since the proofs for the two inequalities are essentially the same, we shall omit the proof of (ii).

Suppose first that $k=0$. From Lemma $1,\left|S_{n}(z)\right|<2 e n^{1 / 2}$ for every point $z$ in the closure of the complement of $K$. Therefore, if $d_{K}(z) \geqq \epsilon$,

$$
\left|S_{n}^{\prime}(z)\right|<\frac{2 e n^{1 / 2}}{d_{K}(z)} \leqq \frac{2 e n^{1 / 2}}{\epsilon}
$$

by the Cauchy inequality for derivatives. Since 1 is in $K,|z-1| \geqq \epsilon$. Therefore, from (ii) of Lemma 1, we have

$$
\left|S_{n}(z)\right|<\left(1+\frac{1}{\epsilon}\right)\left(1+\frac{2 e n^{-1 / 2}}{\epsilon}\right)<\frac{14}{\epsilon^{2}},
$$

since $\epsilon<1$.

Suppose now that $k$ is an integer for which (i) is true for every positive $\epsilon$ less than 1 . Let

$$
F(z)=\left(\frac{z}{z-1} \frac{d}{d z}\right)^{k} S_{n}(z)
$$

Then for all $z$ for which $d_{K}(z) \geqq \epsilon / 2$,

$$
|F(z)|<14 \frac{2^{k^{2}+3 k}}{(\epsilon / 2)^{2 k+2}}=14 \frac{2^{k^{2}+5 k+2}}{\epsilon^{2 k+2}} .
$$

Then by the Cauchy inequality, we have, for all $z$ such that $d_{K}(z) \geqq \epsilon$,

$$
\left|F^{\prime}(z)\right|<14 \frac{2^{k^{2}+5 k+3}}{\epsilon^{2 k+3}} .
$$

Since

$$
\left|\frac{z}{z-1}\right| \leqq 1+1 / \epsilon<2 / \epsilon
$$

we have

$$
\left|\left(\frac{z}{z-1} \frac{d}{d z}\right) F(z)\right|<14 \frac{2^{k^{2}+5 k+4}}{\epsilon^{2 k+4}} .
$$

Consequently, by mathematical induction, (i) is true for every nonnegative integer $k$. This completes the proof.

If for each nonnegative integer $k$ we let 


$$
U_{k}(z)=\left(\frac{z}{z-1} \frac{d}{d z}\right)^{k} \frac{z}{1-z},
$$

then for the first few values of $k$ we have

$$
\begin{aligned}
& U_{0}(z)=\frac{z}{1-z}, \quad U_{1}(z)=\frac{z}{(z-1)^{3}}, \\
& U_{2}(z)=(-1) \frac{z+2 z^{2}}{(z-1)^{5}}, \quad U_{8}(z)=\frac{z+8 z^{2}+6 z^{3}}{(z-1)^{7}}, \\
& U_{4}(z)=(-1) \frac{z+22 z^{2}+58 z^{3}+24 z^{4}}{(z-1)^{9}} \text {, }
\end{aligned}
$$

and, in general,

$$
U_{k}(z)=\frac{(-1)^{k+1} Q_{k}(z)}{(z-1)^{2 k+1}},
$$

where, for $k>1, Q_{k}(z)$ is a polynomial of degree $k$ with positive integer coefficients.

We are now in a position to state our principle result, the proof of which follows immediately from Lemmas 2 and 3.

Theorem. Suppose $\epsilon>0$. For every positive integer $k$, the asymptotic formulas

$$
S_{n}(z)=\sum_{r=0}^{k-1}\left(\frac{1}{n}\right)^{r} U_{r}(z)+O\left(n^{-k}\right)
$$

and

$$
T_{n}(z)=-\sum_{r=0}^{k-1}\left(\frac{1}{n}\right)^{r} U_{r}(z)+O\left(n^{-k}\right)
$$

hold uniformly for $d_{K}(z) \geqq \epsilon$ and $d_{J}(z) \geqq \epsilon$, respectively.

Since every point except $z=1$ belongs either to the complement of $J$ or to the complement of $K$, we can make use of (i) of Lemma 1 and the characteristic functions $\phi_{J}(z)$ and $\phi_{K}(z)$ to combine (3) and (4) and obtain the following:

CoRollary. If $z \neq 1$, then $S_{n}(z)$ and $T_{n}(z)$ have the asymptotic expansions

$$
S_{n}(z) \sim n !\left(\frac{e^{z}}{n z}\right)^{n} \phi_{K}(z)+\sum_{r=0}^{\infty}\left(\frac{1}{n}\right)^{r} U_{r}(z), \text { and }
$$




$$
T_{n}(z) \sim n !\left(\frac{e^{z}}{n z}\right)^{n} \phi_{J}(z)-\sum_{r=0}^{\infty}\left(\frac{1}{n}\right)^{r} U_{r}(z) .
$$

If $z=-1$, (5) reduces to Copson's series. Ramanujan's approximation for $S_{n}(1)$ is, in view of (5), a considerably more singular result than it would otherwise appear.

\section{REFERENCES}

1. E. T. Copson, An approximation connected with $e^{-x}$, Proc. Edinburgh Math. Soc. (2) 3 (1932-33), 201-206.

2. S. Ramanujan, Collected papers, p. 26, Cambridge, 1927.

3. G. Szegö, Über einige von S. Ramanujan gestellte Aufgaben, J. London Math. Soc. 3 (1928), 225-232.

4. G. N. Watson, Approximations connected with $e^{x}$, Proc. London Math. Soc. (2) 29 (1928-29), 293-308.

University of North Carolina

\section{ON THE LOCAL LINEARIZATION OF DIFFERENTIAL EQUATIONS ${ }^{1}$}

\section{PHILIP HARTMAN}

1. Consider the autonomous system of real, nonlinear differential equations

(1.1) $x^{\prime}=E x+F(x)$, where $F(|x|)=o(|x|)$ as $x \rightarrow 0$,

$x$ is a (Euclidean) vector, $F(x)$ a smooth vector-valued function of $x$, and $E$ a constant matrix with eigenvalues $e_{1}, e_{2}, \cdots$ satisfying

$$
\operatorname{Re} e_{j} \neq 0 \text {. }
$$

Let the solution $\xi(t, x)$ of (1.1) starting at $x$ for $t=0$ be written as

$$
T^{t}: x^{t}=\xi(t, x)=e^{E t} x+X(t, x),
$$

where $X(t, x)=o(|x|)$ as $x \rightarrow 0$ (for fixed $t$ ). Thus if $T^{t}$ is considered as a map $x \rightarrow x^{t}$, for fixed $t$, the composition rule

$$
T^{t} T^{s}=T^{t+8}
$$

is valid for small $|x|$. Correspondingly, the linear system

Received by the editors May 26, 1962.

${ }_{1}^{1}$ This research was supported by the Air Force Office of Scientific Research. 